\title{
Lung cancer screening: current status
}

\author{
Theresa C. McLoud
}

Received: 27 October 2013/Accepted: 4 November 2013/Published online: 22 November 2013

(C) Italian Society of Medical Radiology 2013

Lung cancer remains the leading cause of death from malignancy with approximately 1.3 million deaths occurring world wide per year [1]. Approximately $70 \%$ of cases have incurable disease at presentation which is either widely metastatic or locally advanced [1]. The overall 5-year survival still remains very low at only $14 \%$ [1]. Lung cancer, however, which is detected early in its course, demonstrates much better survival data. Survival in non-small-cell lung cancer Stage I is greater than $70 \%$ and small peripheral less than $1 \mathrm{~cm}$ lung cancers have survival rates of greater than $80 \%$ [2]. The rationale for screening is, therefore, obvious. Diagnosis of lung cancer early in preclinical stages has a higher cure rate and, therefore, high-risk asymptomatic individuals are likely to benefit from screening.

The introduction of helical and subsequently multidetector CT in the 1990s led to a number of feasibility studies in which CT was used as a screening tool for the detection of early asymptomatic lung cancer. Observational single arm trials were conducted for several years in many countries including the United States, Japan, and Europe [3-8]. These studies established the sensitivity of low-dose CT for early lung cancer detection. These trials also tested algorithms for diagnostic workup and demonstrated a reduction in the incidence of advanced non-small-cell lung cancer with screening. A high percentage (84-93\%) was Stage I lung cancers [3-5]. They also produced somewhat similar results. They demonstrated that detection of earlystage lung cancer was higher than that reported previously with standard chest radiography [9] but that the false

T. C. McLoud ( $\square)$

Thoracic Radiology, Massachusetts General Hospital, Harvard Medical School, 55 Fruit Street, Boston, MA 02114, USA

e-mail: tmcloud@partners.org positive rate was at least three times that of standard radiographs. In regard to diagnostic algorithms, these studies provided evidence that peripheral nodules greater than $8-10 \mathrm{~mm}$ in size required further investigation because of the high likelihood of lung cancer. Such investigations included further imaging with PET-CT, percutaneous biopsy or resection and often led to invasive procedures. Smaller nodules $(<8 \mathrm{~mm}$ diameter) could be managed with $\mathrm{CT}$ follow-up to determine interval growth indicating the likelihood of malignancy.

These single arm feasibility and observational studies provided compelling evidence of the effectiveness of helical and MDCT as a screening tool for early detection of lung cancer. However, they were not sufficient to determine screening effectiveness because they were not able to assess the influence of CT screening on lung cancer mortality. Only randomized controlled trials can determine effectiveness of screening where the end point must be a decrease in mortality in the screened group as compared to a non-screened control.

Subsequently, several prospective randomized controlled trials were undertaken both in the United States and Europe. These included the National Lung Cancer Screening Trial (NLST) in the United States [10] and the Nelson Trial and others in Europe [11-15]. The results of the NLST trial were published in 2011 [16]. The trial was a cooperative effort between the National Cancer Institute of the NIH and ACRIN, the American College of Radiology Imaging Network. 53,454 individuals were enrolled in the trial. It was a randomized national study and the population group included individuals between the ages of 55 and 74 with history of $\geqq 30$-pack years of current or prior smoking. The enrollees underwent three annual screens. The study group received low-dose CT and the control group underwent standard chest radiography. CT was conducted with 
low dose (1.5 mSv) helical or multidetector CT. The study was designed to determine lung cancer-specific mortality and had a $90 \%$ power to detect a $20 \%$ reduction in lung cancer in the screened group. A positive screen consisted of the detection of a nodule or nodules greater than $4 \mathrm{~mm}$ in diameter or other abnormalities suspicious of lung cancer. Follow-up was recommended based on nodule size and larger nodules required diagnostic workup. A negative screen consisted of the absence of nodules or nodules less than $4 \mathrm{~mm}$ in diameter with no other or abnormality suggestive of lung cancer. Results reported in 2011 indicated a $20 \%$ reduction in mortality in the $\mathrm{CT}$ arm as well as an overall mortality reduction from all causes of $6.7 \%$. More lung cancers were detected with CT than standard radiography and a true stage shift was observed in the CT arm with more lung cancers identified in earlier stages. There were few major complications from diagnostic workups conducted on cases suspicious for lung cancer.

However, there were a number of important disadvantages of screening reported by this study. The first was the high positivity rate. $39 \%$ of subjects in the CT arm had a positive scan over the course of the 3 -year screening period and $95 \%$ of the positive screens were false positives with no evidence of confirmed lung cancer. The second disadvantage of CT screening was the exposure of patients to radiation. However, the technique delivered a mean whole body effective dose of $1.4 \mathrm{mSv}$ in the NLST trial which represents about one-fifth of the dose of a diagnostic chest CT scan [16].

The potential benefits of CT screening for high-risk individuals outweigh the small risk of cancer deaths related to cumulative radiation exposure [17].

There are methods to improve the positive predictive value and decrease the large number of false positives encountered with CT screening for lung cancer. The NLST interpretation algorithm was dichotomous. The Nelson Trial in Europe is a two-step process which has been able to decrease the number of false positive findings based on nodule size [11]. Nodules less than 4-6 $\mathrm{mm}$ are considered negative and continue with the incidence screens. Those greater than approximately $1 \mathrm{~cm}$ are considered positive and undergo a definitive workup. There is an indeterminate group consisting of nodules between 6 and $10 \mathrm{~mm}$ in diameter. If there is a finding of a nodule in this range on the prevalence screen, individuals undergo a 3-month follow-up. The study is considered positive if there is a greater than $25 \%$ growth and these patients undergo definitive workup. The negative group undergoes an annual screening CT. This approach has improved the positive predictive value to $36 \%$ in the prevalence scan and $42 \%$ on the incidence scan. Another method of improving the positive predictive value is to better define risk profiling. Although the greatest risk for lung cancer is smoking, the risk is less than $20 \%$ for the development of lung cancer. However, patients with COPD may have up to a sixfold increased risk for malignancy. It is also possible that medical biomarkers may be used to further identify highrisk populations.

Many American professional societies have recommended lung cancer screening for individuals meeting the criteria of the NLST trial with some slight modifications. These include the American College of Chest Physicians, the American Cancer Society, the American Association of Thoracic Surgery, and the National Comprehensive Cancer Network [17, 18]. More recently, the United States Preventative Services Task Force (USPSTF) has developed a draft recommendation (Grade B) for CT lung cancer screening of high-risk patients (those 55 to 79 years old with 30-pack/years or greater history of smoking) (http:// www.uspreventativeservicetaskforce.org/draftrec.htm). In the United States, the Affordable Care Act requires that CMS (government healthcare) and private insurers provide coverage of all medical exams or procedures that receive a Grade of B or higher from USPSTF. This will ultimately mean that in the US, there will be insurance coverage for lung cancer screening probably within the course of a year.

The cost-effectiveness data from the NLST trial have not yet been published. Estimates of the cost-effectiveness of screening CT have relied on decision analysis modeling with estimates ranging from $\$ 50,000$ up to $\$ 2,000,000$ per quality-adjusted life year gained [19].

It is extremely important for institutions to develop programs for lung cancer screening. Screening clinics should be formed that provide a transdisciplinary approach among cancer specialists ideally in institutions that have experience in screening and in which protocols are well defined. The American College of Radiology is developing appropriateness criteria and practice guidelines for lung cancer CT screening. Radiologists will require improved technology such as computer-assisted detection of pulmonary nodules (CAD) and volumetric software for lung cancer detection. Future challenges include education of radiologists and management of the large workflow. In Europe, there has been much discussion of the possibility of training physician extenders (such as technologists or radiographers), to provide the initial interpretation of screening examinations. This is probably unlikely to occur in the US because of the degree of healthcare regulation.

Widespread screening will present unique challenges for the specialty of radiology. Evidence-based infrastructure must be in place to secure that patients have access to uniform quality care and a similar life saving benefit from CT examinations that was demonstrated in the National Lung Cancer Screening Trial. Our challenge is to develop robust screening programs that are necessary to provide such care. 
Conflict of interest The author, Theresa McLoud declares no conflict of interest in regard to the contents of this manuscript.

\section{References}

1. Siegel R, Naishadham D, Jemal A (2012) Cancer statistics, 2012. CA Cancer J Clin 62(1):10-29

2. National Cancer Institute (2012) Non-small cell lung cancer treatment (PDQ). Stage 1 non-small lung cancer. Updated 2/10/ 12. http:/www.cancer.gov/cancertopics/pdq/treatment/non-smallcell-lung/HealthProfessional/page8

3. Kaneko M, Eguchi K, Ohmatsu H et al (1996) Peripheral lung cancer: screening and detection with low-dose spiral CT vs. radiography. Radiology 201(3):798-802

4. Sone S, Takashima S, Li F et al (1998) Mass screening for lung cancer with mobile spiral computed tomography scanner. Lancet 351(9111):1242-1245

5. Henschke CL, McCauley DL, Yankelevitz DF et al (1999) Early Lung Cancer Action Project: overall design and findings from baseline screening. Lancet 354(9173):99-105

6. Swensen SJ, Jett JR, Hartman TE et al (2005) CT screening for lung cancer: 5-year prospective experience. Radiology 235(1): 259-265

7. Nawa T, Nakagawa T, Kusano S, Kawasaki Y, Sugawara Y, Nakata H (2002) Lung cancer screening using low-dose spiral CT; results of baseline and 1-year follow-up studies. Chest 122(1):15-20

8. Diederich S, Wormanns D, Semik M et al (2002) Screening for early lung cancer with low-dose spiral CT; prevalence in 817 asymptomatic smokers. Radiology 222(3):773-781

9. Fontana RS, Sanderson DR, Woolner LB et al (1986) Lung cancer screening: the Mayo program. J Occup Med 28(8): $746-750$

10. Aberle DR, Berg CD, Black WC et al (2011) National Lung Cancer Screening Trial Research Team. The National Lung
Screening Trial: overview and study design. Radiology 258(1):243-253

11. van Lersel CA, de Koning HJ, Draisma G et al (2007) Risk-based selection from the general population in a screening trial: selection criteria, recruitment and power for the Dutch-Belgian randomized lung cancer multi-slice CT screening trial (NELSON). Int J Cancer 120(4):868-874

12. Pedersen JH, Ashraf H, Dirksen A et al (2009) The Danish randomized lung cancer CT screening trial-overall design and results of the prevalence round. J Thorac Oncol 4(5):608-614

13. Lopes Pegna A, Picozzi G, Mascalchi M, ITALUNG Study Research Group et al (2009) Design, recruitment and baseline results of the ITALUNG trial for lung cancer screening with lowdose CT. Lung Cancer 64(1):34-40

14. Infante M, Cavuto S, Lutman FR et al (2009) DANTE Study Group. A randomized study of lung cancer screening with spiral computed tomography: 3-year results from the DANTE trial. Am J Respir Crit Care Med 180(5):445-453

15. Becker N, Delorme S, Kauczor H-U (2008) LUSI: the German component of the European trial on the efficacy of multi-slice CT for the early detection of lung cancer (abstract). Onkologie 31(suppl 1):P320

16. Aberle DR, Adams AM, Berg CD et al (2011) National Lung Screening Trial Research Team. Reduced lung-cancer mortality with low-dose computed tomographic screening. N Engl J Med 365(5):395-409

17. Bach PB, Mirkin JN, Oliver TK et al (2012) Benefits and arms of CT screening for lung cancer: a systematic review. JAMA 307(22):2418-2429

18. Wood DE, Eapen GA, Ettinger DS et al (2012) Lung cancer screening. J Natl Compr Canc Netw 10(2):240-265

19. Wisnivesky JP, Mushlin AI, Sicherman N et al (2003) The costeffectiveness of low-dose CT screening for lung cancer: preliminary results of baseline screening. Chest 124(2):614-621 